\title{
O ESTADO DA ARTE SOBRE ÉTICA EM SAÚDE NO BRASIL: PESQUISA EM BANCO DE TESES
}

\author{
Magda Santos Koerich ${ }^{1}$, Alacoque Lorenzini Erdmann ${ }^{2}$
}

\footnotetext{
${ }^{1}$ Doutora em Enfermagem. Professora Adjunto do Departamento de Patologia da Universidade Federal de Santa Catarina (UFSC). Santa Catarina, Brasil. E-mail: mskoerich@ccs.ufsc.br

${ }^{2}$ Doutora em Filosofia da Enfermagem. Professora Titular do Departamento de Enfermagem e do Programa de Pós-Graduação em Enfermagem da UFSC. Pesquisadora CNPq. Coordenadora de Área de Enfermagem na CAPES. Santa Catarina, Brasil. E-mail: alacoque@newsite.com.br
}

RESUMO: O objetivo foi identificar as abordagens teóricas utilizadas para ensinar ética nos cursos da área da saúde. Trata-se de pesquisa bibliográfica que analisou resumos de teses defendidas entre 1994 e 2009, disponíveis no banco de teses da CAPES, totalizando 721 teses relacionadas ao tema. Com o descritor ensino de ética foram encontrados 345 estudos oriundos, principalmente, da Educação. O descritor ética em saúde permitiu a análise de 393 teses, destacando os Programas de Pós-Graduação em Enfermagem e em Saúde Coletiva. Com o descritor ética, educação e saúde foram analisadas 83 teses, especialmente na Educação. A ética é tema atual, estudada sob múltiplos referenciais, destacando responsabilidade, bioética, relações, cuidado e compreensão. A pesquisa em banco de teses permitiu um recorte da rede de conhecimentos sobre ética em saúde e mostrou a necessidade de mais estudos mediante a diversidade de referenciais teóricos, metodológicos e paradigmáticos e pelas especificidades próprias dos agentes envolvidos.

DESCRITORES: Pesquisa em enfermagem. Coleta de dados. Revisão ética. Recursos humanos em saúde. Estudantes de ciências da saúde.

\section{THE STATE OF ART ON ETHICS IN BRAZILIAN HEALTH SCIENCES: RESEARCH IN DISSERTATION DATABASE}

\begin{abstract}
The goal of this bibliographic study was to identify the theoretical approaches used to teach ethics in the health sciences area. In order to do so, abstracts of dissertation defended between 1994 and 2009, available in CAPES dissertation database, with 721 dissertation related to the theme were analyzed. The descriptor "teaching ethics" resulted in 345 studies originating mainly from Education. The descriptor "ethics in health" permitted the analysis of 393 dissertation, highlighting Nursing Graduate Programs and Collective Health. With the descriptor "ethics, education, and health", 83 dissertation were analyzed, especially from Education. Ethics is an up-to-date issue, now being studied from multiple references, highlighting issues such as: responsibility, bioethics, relationships, care, and understanding. Researching within the dissertation database allowed for a cross section of the knowledge network in ethics in health sciences and showed the need for further study in facing the diversity of theoretical, methodological, and paradigmatic references and the specific characteristics of those involved.
\end{abstract}

DESCRIPTORS: Nursing research. Data collection. Ethics review. Human resources in health. Health sciences students.

\section{EL ESTADO DEL ARTE DE LA ÉTICA EN SALUD EN BRASIL: INVESTIGACIÓN EN UNA BASE DE DATOS DE TESIS}

\begin{abstract}
RESUMEN: El objetivo fue identificar los enfoques teóricos utilizados para enseñar la ética en los cursos del área de la salud. Se trata de una investigación bibliográfica que examinó los resúmenes de las tesis defendidas entre 1994 y 2009, disponibles en la base de datos de tesis de la CAPES, para un total de 721 tesis relacionadas con el tema objeto del estudio. El descriptor: la enseñanza de la ética está presente en 345 estudios, principalmente del área de Educación. El descriptor: la ética en la salud permitió el análisis de 393 tesis, destacando los programas de postgrado en Enfermería y de Salud Colectiva. Con el descriptor: ética, educación y salud se analizaron 83 tesis, especialmente en Educación. La ética es un tema actual que se estudia desde múltiples referencias y señala cuestiones tales como: responsabilidad, bioética, relaciones, cuidado y comprensión. La investigación en base de datos permitió un recorte de la red de conocimientos sobre la ética en la salud y mostró la necesidad de más estudios por la diversidad de enfoques teóricos, metodológicos y paradigmáticos, y por las características de los agentes involucrados.
\end{abstract}

DESCRIPTORES: Investigación en enfermería. Recolección de datos. Revisión ética. Recursos humanos en salud. Estudiantes del área de la salud. 


\section{INTRODUÇÃO}

A palavra grega éthos, da qual se originou a ética, relaciona-se à conduta, hábito ou comportamento. Possui também os sentidos de: morada do homem (oikos), seu gênio protetor (daímon), regido pelo logos; modo de proceder do homem como dono de seus hábitos adquiridos pela repetição e não regidos pela natureza (physis); refere-se, ainda, à liberdade, sob a soberania da lei justa. ${ }^{1}$

Apresentada como necessária à democracia e ao convívio em sociedade, desde a antiguidade, a ética tem sido preocupação e ocupação de muitos pensadores que apresentam propostas e significados similares ou contraditórios.

$\mathrm{Na}$ atualidade, a ética novamente ocupa um lugar de destaque, não apenas como campo do saber acadêmico, mas também no cotidiano da vida social, profissional e pessoal, no meio científico, político, econômico, nas áreas da educação e da saúde. Pode ser compreendida como uma reflexão filosófica sobre a moralidade ou simplesmente como a ciência da conduta. ${ }^{2-3}$ Pode ainda apresentar-se como uma emergência a partir da razão e emoção que define a forma como uma pessoa faz suas escolhas. ${ }^{4}$

Descortina-se um cenário de múltiplas abordagens e de contínuo interesse geral pela temática da ética, mas que permanece como constante inquietação, gerando alguns questionamentos: o que ainda é possível apreender sobre ética? Por quê a ética? Como abordar a ética como tema de pesquisa e torná-la um tema original e inédito?

Como as formas e estilos de olhar o mundo são múltiplos, é possível e, muito provável, que cada pesquisador, ao investigar e analisar um mesmo objeto, perceba diferentes nuanças a seu respeito ou semelhanças em relação ao que já foi construído por outros pesquisadores. Dessa forma, ao observarmos um determinado objeto, podemos descrevê-lo apenas a partir do nosso ângulo de visão ou variando as posições, de baixo para cima ou de cima para baixo ou, ainda, com mais ou menos luminosidade. Podemos aumentar ou diminuir a distância que nos separa desse objeto. Podemos também trocar experiências com outros observadores e, assim, estaremos abrindo novas possibilidades de ver e representar, de formas muito variadas, o objeto/mundo que vemos e nos propomos a descrever.

Assim, a busca pela originalidade e ineditismo do tema de pesquisa passa a ser secundária, visto que os construtos elaborados apresentam-se como expressão da subjetividade do pesquisador e dos sujeitos da pesquisa, num determinado contexto e momento histórico. São, portanto, originais e únicos.

No caso específico da ética, por ser um tema recorrente, que tem subsidiado estudos em várias áreas, as pesquisas denominadas estado da arte são extremamente úteis quando o pesquisador pretende construir conhecimentos com características de subjetividade, mas também de originalidade e ineditismo.

O objetivo desse estudo foi identificar as diversas abordagens teóricas utilizadas para ensinar ética nos cursos da área da saúde, a partir das informações do banco de teses da CAPES.

\section{MÉTODO}

As pesquisas denominadas "estado da arte" ou "estado do conhecimento", referem-se a pesquisas bibliográficas, com a pretensão de "mapear e de discutir certa produção acadêmica em diferentes campos do conhecimento, tentando responder que aspectos e dimensões vêm sendo destacados e privilegiados em diferentes épocas e lugares". .:257 Utilizam-se, geralmente, os resumos a partir de dissertações, teses, publicações em periódicos e em anais de congressos e de outros eventos científicos.

Ao buscar informações nos trabalhos já publicados, é possível perceber a presença de múltiplos enunciados, de influências teóricas próprias, da compreensão alcançada pelos autores e das possíveis relações e contradições. Forma-se, assim, uma "rede de vários fios que se cruzam, se rompem, se unem, se questionam dependendo do ponto que se estabelece como partida em cada texto" ${ }^{\text {5:270 }}$

Foram analisados os resumos de teses disponibilizados no Banco de Teses da Coordenação de Aperfeiçoamento de Pessoal de Nível Superior (CAPES), entre os anos de 1994 e 2009 inclusive. A partir dos descritores ensino de ética, ética em saúde, e ética, educação e saúde, foram verificados os títulos e resumos de todas as teses e selecionadas aquelas que se adequavam aos seguintes critérios de inclusão: a) estudos que se sustentaram no pensamento complexo de Edgar Morin e na razão sensível de Michel Maffesoli; b) estudos fundamentados por referenciais teóricos da ética ou bioética e; c) os estudos relacionados ao ensino de ética nos cursos de nível superior na área da saúde. 


\section{RESULTADOS E DISCUSSÃO}

Foram encontradas 721 teses em que se verifica o interesse crescente pelo tema da ética, com aumento significativo de trabalhos a partir de 2002, destacando-se os anos de 2007 com 103 teses defendidas e 2009 com 130 , conforme os resultados apresentados na tabela 1 , a seguir.

Tabela 1 - Número de teses defendidas por ano e por descritor, armazenadas pela CAPES

\begin{tabular}{|c|c|c|c|c|c|c|c|c|c|c|c|c|c|c|c|c|c|}
\hline \multirow[b]{2}{*}{ Descritores } & \multicolumn{17}{|c|}{ Ano de defesa da tese } \\
\hline & $\stackrel{+}{\circ}$ & 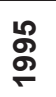 & $\stackrel{\circ}{\circ}$ & ㅎํㅇ & $\stackrel{\infty}{\stackrel{\circ}{\circ}}$ & $\stackrel{9}{\circ}$ & ঃั & ণัे & ัิ & ర్రి & ষ্ণ & น̊ำ & ๕ั & ڤ્ণ & ঃ̊ & ㅇํํ & ฮٓ \\
\hline Ensino de ética & 1 & 2 & 3 & 4 & 5 & 12 & 15 & 16 & 24 & 24 & 32 & 31 & 36 & 42 & 44 & 54 & 345 \\
\hline Ética em saúde & - & 5 & 1 & 6 & 6 & 13 & 14 & 17 & 28 & 28 & 36 & 24 & 55 & 51 & 49 & 60 & 393 \\
\hline Ética, educação e saúde & - & - & - & - & 2 & 2 & 3 & 3 & 6 & 9 & 17 & 6 & - & 10 & 9 & 16 & 83 \\
\hline Total & 1 & 7 & 4 & 10 & 13 & 27 & 32 & 36 & 58 & 61 & 85 & 61 & 91 & 103 & 102 & 130 & 721 \\
\hline
\end{tabular}

O descritor "ensino de ética" foi utilizado em 345 estudos, quase todos oriundos da área da educação e voltados, principalmente, ao ensino fundamental, como orientação aos professores das fases iniciais.

Em relação à educação superior, destacaram-se a abordagem de novos paradigmas para embasar o ensino em nível terciário e a necessidade de desenvolver o senso crítico dos estudantes universitários.

Observa-se um aumento significativo de estudos sobre ética na área da saúde a partir de 2007, especialmente na enfermagem, medicina e odontologia. Informação comprovada no estudo que analisou publicações em periódicos nacionais, de 1997 a 2009, onde foi constatado que dentre as 14 profissões da área da saúde, haviam produções de apenas quatro delas: medicina, enfermagem, odontologia e psicologia. ${ }^{6}$

Com o descritor "ética em saúde" foram encontrados 393 trabalhos, com destaque para o ano de 2006, com 55 teses defendidas sobre o tema e de 2009, com 60 trabalhos. Um dado que chama atenção refere-se às áreas de enfermagem e saúde coletiva que desenvolveram uma expressiva quantidade de trabalhos, seguidos pela medicina, a psicologia e a odontologia. Essa constatação não coincide com o número de publicações em periódicos, em que a medicina ocupa o primeiro lugar com maior quantidade de artigos, seguida pela Enfermagem e saúde coletiva. ${ }^{6}$ Isso demonstra que o interesse pelo tema da ética não é semelhante para todos os cursos da saúde, sendo necessário investir mais nessa questão. Em outras áreas como educação, direito, sociologia e ciências da saúde também foram encontrados alguns estudos relacionados ao descritor.

O descritor "ética, educação e saúde" contemplou 83 teses, sendo que algumas coincidiram com os descritores anteriores. Neste destacaram-se trabalhos na área da educação, seguida pela enfermagem e odontologia.

O conjunto de trabalhos relacionados à ética e formação ética dos profissionais da saúde permitiram seu agrupamento nas seguintes categorias: a) Formação ética dos profissionais da saúde a partir da emergência de várias éticas; b) Formação ética dos profissionais da saúde sob os referenciais teóricos de Edgar Morin e Michel Maffesoli; c) Formação ética dos profissionais da saúde sob a ótica de outras ciências/referenciais.

\section{Formação ética dos profissionais da saúde a partir da emergência de várias éticas}

A ética se coloca como tema polissêmico, com múltiplos sentidos e aplicações. Assim, podemos apreciar seu caráter normativo, na ética aplicada para resolução de problemas éticos cotidianos, ou seu caráter reflexivo, também como ética utilitarista, ou ainda, a deontológica, além das diversas escolas filosóficas onde a ética se origina. $^{7}$

Assim, pode ser uma questão ética o desenvolvimento do senso crítico entre os estudantes universitários, na intenção de capacitá-los para que efetuem bons julgamentos, ou seja, juízos críticos fundamentados na observação objetiva dos fatos, que considerem aspectos éticos e capacidade empática de compreensão da realidade alheia. ${ }^{8}$ 
$\mathrm{Na}$ enfermagem, destaca-se o estudo que coloca a ética fundamentada nos princípios da bioética, como pressuposto fundamental do seu saber. ${ }^{9} \mathrm{Um}$ trabalho analisou a fundamentação dos juízos morais de enfermeiros sobre o direito à informação e a iatrogenia cirúrgica como dilemas morais enfrentados na prática assistencial ${ }^{10}$, enquanto outros dois autores avaliaram a aprendizagem em enfermagem na área de saúde da mulher e saúde psiquiátrica, respectivamente; ambos voltados para a dimensão ética. ${ }^{11-12}$ Em um estudo quantitativo com enfermeiros, foram estabelecidos "pontos de luz e buracos negros" na formação desses profissionais, destacando carências quanto à formação do profissional crítico, reflexivo, capaz de atuar e promover mudanças. ${ }^{13}$

A partir do referencial de poder de Michel Foucault, a saúde das pessoas pode ser focalizada como responsabilidade da enfermagem, sendo que o cliente ocupa o lugar de objeto do cuidado. O cuidado de si é abordado como uma preocupação ética e como objetivo da enfermagem para o exercício da autonomia do cliente, sujeito a fim da saúde. ${ }^{14}$

Por outro lado, resgata-se as possibilidades de realização subjetiva do trabalho em saúde, a partir de um marco sociológico das relações de trabalho e subjetividade; de um marco do próprio trabalho em saúde e de um marco filosófico que aponta o entrelaçamento entre ética e estética com o trabalho. ${ }^{15}$

A educação em saúde foi abordada junto a um grupo de usuários de saúde, na perspectiva da bioética, revelando condutas profissionais autoritárias e paternalistas, que não consideram o consentimento informado e fomentam atitudes passivas e de dependência, características estas que evidenciam falta de respeito pela pessoa, sua dignidade e sua autonomia. ${ }^{16}$ Ainda sobre a bioética, apresenta-se a solidariedade crítica como valor e uma analisse do principialismo da bioética na atenção básica. ${ }^{17-18}$

As novas tecnologias reprodutivas são discutidas como a última etapa de um processo contínuo de medicalização da sexualidade e da reprodução, em que se apresenta o debate ético em torno da temática, travado por especialistas, o qual termina por produzir uma verdadeira medicalização da própria ética, conduzindo a novos impasses que se desdobram de forma bem mais ampla, para além do campo da reprodução biológica. ${ }^{19}$

A ética da responsabilidade, do pensador Hans Jonas, é apresentada em um estudo com profissionais da saúde relacionado à prudência no uso de antibióticos. ${ }^{20} \mathrm{Na}$ enfermagem a ética da responsabilidade se constitui uma referência para o cuidado. A reflexão acerca da ética da responsabilidade deve incluir perspectivas políticas da sociedade e da profissão, ultrapassa a ética deontológica e pode ser visualizada no apoio dado ao usuário, na busca pela melhoria da qualidade dos serviços e na cooperação interprofissional e intersetorial. "A responsabilidade produz efeitos integrativos da dignidade humana, ponderando circunstâncias concretas do cuidar e tomar decisões razoáveis, prudentes e equitativas." 7:80

Em relação a atitude decisória, afirma-se que o modo como os trabalhadores da saúde tomam suas decisões tem implicações morais importantes que podem repercutir direta ou indiretamente no cuidado do usuário. Destaca-se, também, que as relações estabelecidas podem favorecer a tomada de decisões e o exercício da autonomia. ${ }^{21}$

Na medicina, merece destaque um estudo, com título bastante sugestivo: "Saindo da adolescência com a vida (dos outros) nas mãos: estudo sobre a formação ética dos estudantes de medicina". Defende o ensino da ética aplicada como disciplina na graduação, inserida no ensino das humanidades médicas, e aponta para a possibilidade de estabelecer seu ensino transversalmente. ${ }^{22}$

Destaca-se também o trabalho que aponta a violência como um grave problema, colocado pela racionalidade médica e que não pode ser abordado, apenas, a partir da ética humanista ou da bioética. ${ }^{23} \mathrm{Com}$ tema similar, em outro estudo refletiu-se sobre a função da confidencialidade para os profissionais de saúde que lidam com pessoas envolvidas em casos de violência, na tentativa de avaliar os limites éticos destas intervenções e constata a dificuldade dos profissionais ao lidarem com tais casos. ${ }^{24}$

Algumas experiências de trabalho em comunidade podem tornar-se espaços de aprendizagens diferenciadas para uma formação acadêmica mais plural e heterogênea. Isto porque os processos de interação entre as pessoas e entre os saberes podem contribuir para o desenvolvimento de aprendizagens plurais, extrapolando o limite das puramente instrumentais/técnico/científicas dos atuais currículos de formação de profissionais de saúde, especialmente, dos cursos de enfermagem, odontologia e medicina. ${ }^{25}$

A evolução dos conceitos de ética se deu através dos tempos, permitindo a identificação de alguns marcos conceituais e históricos que ca- 
racterizam diferentes momentos da ética geral e sua evolução para uma ética direcionada para as ciências médicas e enfim, para a bioética. Diante do contexto de mudanças no ensino superior, do avanço científico e tecnológico e do próprio campo da ética e bioética, o tema merece mais atenção no sentido de se conhecer como os cursos de graduação vêm acompanhando essas mudanças e de que forma isto tem se aplicado e influenciado a educação na área da saúde. ${ }^{6}$

Em sua origem no Brasil as discussões éticas, no campo da enfermagem, têm se pautado numa postura que pode ser caracterizada como conservadora por privilegiar aspectos como a religiosidade, autoritarismo, obediência, hierarquia, subserviência e outras. ${ }^{26}$

\section{Formação ética dos profissionais da saúde sob os referenciais teóricos de Edgar Morin e Michel Maffesoli}

O sentido da palavra e do conceito de saúde permite diversas perspectivas de leitura de acordo com diferentes contextos e formas de olhar o mundo ou paradigmas. Da mesma maneira, a formação ética dos profissionais da saúde se insere em perspectivas paradigmáticas, gerando indagações como: qual é a concepção de saúde? Como ensinar ética, mediante tal concepção?

A concepção de saúde ou de ética é fundada em paradigmas teóricos que precisam ser evidenciados para compreender e utilizar a palavra saúde e/ou ética e permitir a interlocução das várias profissões da saúde. ${ }^{27}$

Daí a escolha da complexidade como paradigma. Pensar de forma complexa é pensar de forma integral, pois o complexo é qualidade do que é tecido junto. Assim, a formação profissional e a construção do conhecimento se fazem a partir de múltiplas dimensões, de múltiplos olhares, de múltiplos fazeres/saberes e permite a concomitância no mesmo processo de ensino-aprendizagem, de conteúdos técnicos e científicos com valores éticos que permitam a convivência interumana. ${ }^{28}$

A discussão paradigmática não se atem apenas à questão biológica mas implica em questões de ordem psíquica, antropológica, social e histórica e destaca, necessariamente, as relações complexas existentes entre esses diferentes aspectos e oposições. ${ }^{27}$

Assim, o descritor ensino de ética destacou dois estudos que apontam para novos paradigmas (como o da complexidade) que vislumbram processos abrangentes de percepção mais sutis da realidade. Referem-se à perspectiva intertransdisciplinar do ensino, com a necessária postura de inteireza e totalidade do ser professor. ${ }^{29-30}$

O estudo apoiado nos pressupostos do Interacionismo Simbólico e na proposta teórico-epistemológica de Michel Maffesoli, procurou compreender o mundo imaginal (interações, imagens, imaginário, símbolos e significados) de ser família saudável no cotidiano, em tempos pós-modernos. Destaca a demanda por profissional híbrido e trabalho intertransdisciplinar. ${ }^{31}$ O mesmo referencial teórico foi utilizado para apreender o jogo da diferença entre as formas de cuidar expressas na prática profissional da enfermagem, que apontaram a marca do racional, bem como a emergência do sensível na ação de cuidar e o interstício de um cotidiano rico em astúcias, transgressões, solidariedade e éticas particulares. $^{32}$

Nossa conduta profissional é marcada pela racionalidade, mas também pelo jogo, pela astúcia e pelas máscaras, que fazem do quotidiano uma permanente luta entre o dever-ser e o desejo de apenas ser-e-estar-juntos, um dilema entre a razão e a sensibilidade, referido por Maffesoli. ${ }^{28}$

\section{Formação ética dos profissionais da saúde sob a ótica de outras ciências/referenciais}

$\mathrm{Na}$ vertente fenomenológica, encontramos um estudo que investigou o processo ensino-aprendizagem de ética, durante a graduação em enfermagem, estabelecendo algumas diretrizes curriculares, pautadas nos direitos humanos e no saber/fazer o que é justo. ${ }^{33}$

Uma tese da área da educação apresenta a cartografia dos cenários da educação e saúde com a emergência dos conceitos de "clínica nômade" e de "pedagogia mestiça". Propõe a combinação das práticas biomédicas com os conhecimentos de outras áreas, a fim de ampliar a autonomia dos usuários dos serviços, a partir de um compromisso ético, estético e político e da ruptura com as visões de mundo advindas da modernidade em favor de concepções mais abertas e heterogêneas. ${ }^{34}$ Outro estudo, também da educação, destaca que a dimensão ética deve ser incorporada por todos os responsáveis pelo ensino durante o processo de formação e inserida nos projetos político-pedagógicos dos cursos de medicina, para aplicação edificante da ciência do cuidar/curar. ${ }^{35}$ 
Um estudo em psicologia, com professores de cursos de fisioterapia, apresentou a atividade desse profissional como uma prática educativa, ética e política destinada ao cuidado humano, ${ }^{36}$ ao passo que uma análise do foco foucaultiano destaca as relações entre sujeito e norma e sujeito e ética, além da medicalização do corpo e da sociedade. ${ }^{37}$

O referencial do pensador Ivan Illich é apresentado ao fazer relações entre a tecnologização da medicina, a medicalização e a autonomia, e é confrontado com várias maneiras possíveis de viver bem e de cuidar da saúde, de forma prudente. ${ }^{38}$

A multidimensionalidade humana é discutida em uma tese na área de ciências sociais, tratando do processo de autoconhecimento envolvido na construção da identidade, além de apresentar estratégias de aprendizagem de uma ética da compreensão para a consciência planetária, fundada no amor e nos princípios de esperança e responsabilidade. ${ }^{39}$

Uma pesquisa quantitativa avaliou a prática médica psiquiátrica, destacando uma forte discordância entre os profissionais quanto à experiência de conflitos éticos. Afirma também, que a estrutura da sensibilidade moral dos médicos psiquiatras brasileiros sofre múltiplas influências em sua relação com elementos epidemiológicos, demográficos e culturais. ${ }^{40}$ Finalmente, uma avaliaçãodas atividades do currículo paralelo, entre estudantes de medicina, considera que adicionam considerável carga de trabalho, de responsabilidades e de estresse aos estudantes, podendo ser fator importante no processo de (de)formação da identidade profissional dos alunos. Essa (de) formação na vida estudantil, perpetuada na vida profissional, pode ser um condicionante adicional para levar os estudantes e médicos a aceitarem, no seu dia a dia, o excesso e as más condições de trabalho, a cuidarem mais dos pacientes do que de si mesmos, à quase ausência de lazer e atividades físicas, à má alimentação, contribuindo para acarretar precoces condições de deterioração de sua saúde física e mental. ${ }^{41}$

As demais áreas do conhecimento apresentam vários estudos, com destaque especial para a tese da educação, intitulada: "A aula universitária, espaço de múltiplas relações, interações, influências e referências: um ninho tecido com muitos fios", que parte da concepção de educação como processo de humanização, em que o diálogo e a ética são princípios fundamentais. Utiliza a sala de aula do curso médico e identifica elementos que se aproximam e elementos que se distanciam da formação humana na integralidade, e revela que a aula universitária se constitui e se institui numa dupla dimensão, regularidade plural e diversidade, as quais, contraditoriamente, produzem uma marca de singularidade no contexto do curso médico. ${ }^{42}$

Ao fazer uma análise do ensino de genética em cursos da área da saúde, verifica-se o limitado alcance da contribuição destas práticas para a formação crítica e responsável dos futuros profissionais, frente às complexas implicações sociais e éticas da moderna genética humana e se propõe um redimensionamento do ensino de genética humana para os futuros profissionais de saúde, no sentido de educá-los para atuarem como mediadores entre seus futuros clientes (o público) e a ciência. ${ }^{43}$

\section{CONSIDERAÇÕES FINAIS}

O avanço técnico e científico dos tempos pós-modernos permitiu a emergência de novos desafios e debates nos campos epistemológicos e ontológicos das ciências. Para apreender de forma mais criativa a realidade que se mostra complexa, é necessário mudar o foco no olhar e na forma de apreender o mundo, isto é, na perspectiva de novos e diferentes paradigmas. É preciso reconstruir padrões culturais, subjetivos e ideológicos de referência para perceber a complexidade do mundo que nos cerca e dos objetos que queremos conhecer.

Assim, sempre que tentamos conhecer e utilizar metodologias de cuidado ou de investigação científica, nos tornamos seus críticos ou defensores, uma vez que as posições a favor ou contra esta ou aquela teoria dependem de como vemos o mundo em que vivemos e em que medida somos originais ou reprodutores de uma percepção pré-fabricada e padronizada dos diversos fenômenos e elementos do ambiente que nos cerca, ou do quanto tememos a variação, o novo, a ousadia de transformar a história.

Ao concluir este breve panorama, restrito apenas às teses e a um único banco de dados, ficou evidente que a pesquisa do estado da arte é bastante valiosa, pois tornou possível perceber a tecitura dos fios que formam a rede de conhecimentos da ética em saúde, com destaque especial para a formação ética dos futuros profissionais da saúde. Constatamos, também, que muito mais conhecimento pode ser produzido acerca dessa temática, mediante a diversidade de referenciais 
teóricos, metodológicos e paradigmáticos, mas, principalmente, porque os agentes envolvidos nas pesquisas apresentam características próprias que lhes conferem especificidades, justificando-se, dessa forma, maior produção de conhecimento e aprofundamento do tema e possibilidade de criar linhas de pesquisa na interseção entre educação e ética.

A preferência pelas técnicas e a insuficiência de profissionais qualificados das diversas áreas do conhecimento revelam carência epistemológica para pensar a saúde e a enfermagem no âmbito da ética. ${ }^{7}$

A abordagem da ética nos cursos da área da saúde precisa transcender a questão pedagógica e não ficar restrita apenas à normatização dos códigos de deontologia das profissões, mas ser construída e exercitada no contexto das necessidades da sociedade, possibilitando uma visão ampliada e abrangente dos desafios éticos da vida contemporânea, envolvendo os múltiplos sentidos do nascer, viver e morrer. ${ }^{44}$

\section{REFERÊNCIAS}

1. Schramm FR. A terceira margem da saúde: ética natural, complexidade, crise e responsabilidade no saber-fazer sanitário. Brasília (DF): UNB, 1996.

2. Minaré RL. Bioética. Parc Estrat [online]. 2002 [acesso 2007 Mai 10]; (16): Disponível em: http:/ / www.cgee.org.br/parcerias/p16.php.

3. Dall'Agnol D. Bioética. Coleção passo-a-passo. Rio de Janeiro (RJ): Jorge Zahar; 2005.

4. Cohen C, Segre M. Breve discurso sobre valores, moral, eticidade e ética. In: Serruya SJ, Motta ML, organizadores. Capacitação para Comitês de Ética em Pesquisa. Brasília (DF): Ministério da Saúde; v1, 2006.

5. Ferreira NSA. As pesquisas denominadas estado da arte. Educ Soc [online]. 2002; 79(23): [acesso 2007 Jun 30]. Disponível em: http:/ / www.scielo.br/pdf/es/ v23n79/10857.pdf

6. Carneiro LA, Porto CC, Duarte SBR, Chaveiro $\mathrm{N}$, Barbosa MA. O ensino da ética nos cursos de graduação da área de saúde. Rev Bras Educ Med [online]. 2010 [acesso 2011 Mai 20]; 34(3):412-21. Disponível em: http://www.scielo. br $/$ scielo.php?script $=$ sci_arttext\&pid $=$ S010055022010000300011\&lng=pt\&nrm=iso

7. Souza ML, Sartor VVB, Prado ML. Subsídios para uma ética da responsabilidade em enfermagem. Texto Contexto Enferm [online]. 2005 Mar [citado 2011 Maio 19]; 14(1):75-81. Disponível em: http:/ / www. scielo.br/scielo.php?script=sci_arttext\&pid=S010407072005000100010\&lng $=\mathrm{pt}$
8. Silva ER. O ponto de partida da argumentação: uma metodologia de ensino voltada ao desenvolvimento do senso crítico [tese]. São Paulo (SP): Universidade de São Paulo. Programa de Pós-Graduação em Educação; 1998.

9. Gomes PC. Ética e enfermagem: relações ou reações? [tese]. São Paulo (SP): Universidade Federal de São Paulo. Programa de Pós-Graduação em Enfermagem; 1999.

10. Coelho LCD. Fundamentação dos juízos morais na enfermagem [tese]. Ribeirão Preto (SP): Universidade de São Paulo. Programa de Pós-Graduação em Enfermagem; 2000.

11. Gurgel AH. Aprendizagem em enfermagem na área de saúde da mulher: evidências nas dimensões ética, social e crítica [tese]. Fortaleza (CE): Universidade Federal do Ceará. Programa de Pós-Graduação em Enfermagem; 2002.

12. Chamma RC. Dilemas éticos encontrados no ensino de enfermagem em saúde mental e psiquiátrica [tese]. São Paulo (SP): Universidade de São Paulo. Programa de Pós-Graduação em Enfermagem; 2002.

13. Gabrielli JMW. Formação do enfermeiro: buracos negros e pontos de luz [tese]. Ribeirão Preto (SP): Universidade de São Paulo. Programa de PósGraduação em Enfermagem; 2004.

14. Lunardi VL. Do poder pastoral ao cuidado de si: a governabilidade na enfermagem [tese]. Florianópolis (SC): Universidade Federal de Santa Catarina. Programa de Pós-Graduação em Enfermagem; 1997.

15. Ramos FRS. Obra e manifesto: o desafio estético do trabalho da saúde [tese]. Florianópolis (SC): Universidade Federal de Santa Catarina. Programa de Pós-Graduação em Enfermagem; 1995.

16. Figueroa AA. A bioética e sua presença na educação para a saúde [tese]. Florianópolis (SC): Universidade Federal de Santa Catarina. Programa de PósGraduação em Enfermagem; 1998.

17. Selli L. Bioética, solidariedade crítica e voluntariado orgânico [tese]. Brasília (DF): Universidade de Brasília. Programa de Pós-Graduação em Ciências da Saúde; 2002.

18. Zoboli ELCP. Bioética e atenção básica: um estudo de ética descritiva com enfermeiros e médicos do programa saúde da família [tese]. São Paulo (SP): Universidade de São Paulo. Programa de PósGraduação em Saúde Pública; 2003.

19. Correa MCDV. A tecnologia à serviço de um sonho: um estudo sobre a reprodução assistida [tese]. Rio de Janeiro (RJ): Universidade do Estado do Rio de Janeiro. Programa de Pós-Graduação em Saúde Coletiva; 1997.

20. Santos NQ. O uso indiscriminado de antibióticos e a ecologia das bactérias antibiótico resistentes associadas à problemática da infecção hospitalar: conhecimento e prática dos profissionais de saúde, à luz da ética da responsabilidade de Hans Jonas 
[tese]. Florianópolis (SC): Universidade Federal de Santa Catarina. Programa de Pós-Graduação em Enfermagem; 2002.

21. Silveira RS. A construção moral do trabalhador de saúde como sujeito autônomo e ético [tese]. Florianópolis (SC): Universidade Federal de Santa Catarina. Programa de Pós-Graduação em Enfermagem; 2006.

22. Rego STA. Saindo da adolescência com a vida (dos outros) nas mãos: estudo sobre a formação ética dos estudantes de medicina [tese]. Rio de Janeiro (RJ): Universidade do Estado do Rio de Janeiro. Programa de Pós-Graduação em Saúde Coletiva; 2001.

23. Spinelli HG. Razão, saúde, violência: a (im)potência da racionalidade médica científica [tese]. Campinas (SP): Universidade Estadual de Campinas. Programa de Pós-Graduação em Saúde Coletiva; 1998.

24. Gobbetti GJ. A função da confidencialidade: bioética e incesto [tese]. São Paulo (SP): Universidade de São Paulo. Programa de Pós-Graduação em Ciências Médicas; 2006.

25. Fagundes NC. Em busca de uma universidade outra: a inclusão de "novos" espaços de aprendizagem na formação de profissionais de saúde [tese]. Salvador (BA): Universidade Federal da Bahia. Programa de Pós-Graduação em Educação; 2003.

26. Santiago MMA, Palácios M. Temas éticos e bioéticos que inquietaram a enfermagem: publicações da REBEn de 1970-2000. Rev Bras Enferm. [online]. 2006 Jun [cited 2011 May 19]; 59(3):349-53. Available from: http:/ / www.scielo.br/scielo.php?script=sci_ arttext\&pid=S0034-71672006000300018\&lng=en

27. Birman J. Os sentidos da saúde. Physis. [online]. 1999 [citado 2011 Mai 19]; 9(1):7-12. Disponível em: http://www.scielo.br/scielo.php?script=sci_ arttext\&pid=S0103-73311999000100001\&lng=pt\& nrm=iso

28. Koerich MS. Formando os futuros profissionais da saúde: potências, fragilidades, convergências e divergências vivenciadas por estudantes e professores no processo de formação ética. [tese]. Florianópolis (SC): Universidade Federal de Santa Catarina. Programa de Pós Graduação em Enfermagem; 2009.

29. Santos Neto E. Educação transpessoal: a dinâmica do pessoal e do transpessoal na ação pedagógica e na formação de professores a partir do pensamento de Stanislav Grof [tese]. São Paulo (SP): Pontifícia Universidade Católica de São Paulo. Programa de Estudos Pós-Graduados em Educação; 1998.

30. Furlan VI. Uma nova suavidade e profundidade... O despertar transpessoal e a (re)educação [tese]. Campinas (SP): Universidade Estadual de Campinas. Faculdade de Educação; 1998.

31. Nitschke RG. Uma viagem pelo mundo imaginal de ser família saudável no cotidiano em tempos pósmodernos - a descoberta dos laços de afeto como caminho [tese]. Florianópolis (SC): Universidade Federal de Santa Catarina. Programa de PósGraduação em Enfermagem; 1999.

32. Urasaki MBM. A interconexão da sensibilidade e da razão no cuidar [tese]. São Paulo (SP): Universidade Federal de São Paulo. Programa de Pós-Graduação em Enfermagem; 2000.

33. Carmo HM. Ensinar e aprender ética na graduação em enfermagem: da conduta latente à manifesta [tese]. São Paulo (SP): Universidade Federal de São Paulo. Programa de Pós-Graduação em Enfermagem; 2004.

34. Ferla AA. Clínica nômade e pedagogia médica mestiça: cartografia de idéias oficiais e populares em busca de inovações à formação e à clínica médicas [tese]. Porto Alegre (RS): Universidade Federal do Rio Grande do Sul. Programa de Pós-Graduação em Educação; 2002.

35. Bedin LP. A dimensão ética na formação dos médicos [tese]. Campinas (SP): Universidade Estadual de Campinas. Faculdade de Educação; 2003.

36. Pontes JF. O fisioterapeuta: sua formação e os indícios de uma prática educativa [tese]. São Paulo (SP): Universidade de São Paulo. Programa de PósGraduação em Psicologia; 2002.

37. Pinheiro CVQ. Norma e cuidado de si: Foucault e a história das práticas de subjetividade [tese]. Rio de Janeiro (RJ): Universidade do Estado do Rio de Janeiro. Programa de Pós-Graduação em Saúde Coletiva; 2002.

38. Nogueira RP. A saúde pelo avesso - uma reinterpretação de Ivan Illich, o profeta da autonomia [tese]. Rio de Janeiro (RJ): Universidade do Estado do Rio de Janeiro. Programa de Pós-Graduação em Saúde Coletiva; 1998.

39. Rosa MSO. Educação e saúde: identidade reencontrada [tese]. São Paulo (SP): Pontifícia Universidade Católica de São Paulo. Programa de Estudos Pós Graduados em Ciências Sociais; 2003.

40. Liboni M. A estrutura da sensibilidade moral dos psiquiatras do Brasil [tese]. São Paulo (SP): Universidade de São Paulo. Programa de PósGraduação em Ciências Médicas; 2005.

41. Tavares AP. O currículo paralelo dos estudantes de medicina da Universidade Federal de Minas Gerais [tese]. Belo Horizonte (MG): Universidade Federal de Minas Gerais. Programa de Pós-Graduação em Ciências Médicas; 2006.

42. Cordeiro TSC. A aula universitária, espaço de múltiplas relações, interações, influências e referências: um ninho tecido com muitos fios [tese]. Recife (PE): Universidade Federal de Pernambuco. Programa de Pós-Graduação em Educação; 2006.

43. Rosa VL. Genética e sociedade: conhecimentos significativos e atitudes sobre a hereditariedade na formação de profissionais da saúde [tese]. Florianópolis (SC): Universidade Federal de 
Santa Catarina. Programa de Pós-Graduação em Educação; 2000.

44. Souza ML, Sartor VVB, Prado ML. Subsídios para uma ética da responsabilidade em enfermagem.
Texto Contexto Enferm [online]. 2005[acesso 2011 Mar 20]; 14(1). Disponível em: http:/ /www.scielo. br/scielo.php?script $=$ sci_arttext\&pid $=$ S010407072005000100010\&lng=pt 